\title{
Exploring the characteristics of the visuospatial Hebb repetition effect
}

\author{
MATHIEU COUTURE and SÉBASTIEN TREMBLAY \\ Université Laval, Québec, Québec, Canada
}

\begin{abstract}
Using the dot task (see Jones, Farrand, Stuart, \& Morris, 1995)—regarded as a good visuospatial analogue of the verbal serial recall task-we examined whether the Hebb repetition effect and its characteristics can be extended to visuospatial material. Classically, the Hebb effect has been associated with serial verbal memory: Repetition of a to-be-remembered sequence of verbal items every third trial markedly improves serial recall of that sequence. In the present study, Hebb effects were observed with visuospatial information, and a direct comparison between verbal and spatial sequence learning revealed that the Hebb repetition effect for visuospatial information shares similar characteristics with its verbal analogue. Our results cast some doubt regarding the parsimony of the view that the classical verbal Hebb effect is driven by a store specialized for phonological information and impose some further constraints on modeling serial memory and implicit sequence learning.
\end{abstract}

The capacity to remember the order of events continues to be a key topic of study within experimental psychology, given that the processing of serial order is thought to underpin a wide range of cognitive functions and behaviors (e.g., Lashley, 1951). In the last 40 years or so, serial recall has been used extensively to investigate memory for order over the short term; however, the study of long-term learning of order information has received less attention. In a seminal study by Donald O. Hebb (1961), participants performed an auditory-verbal serial recall task in which one particular series of digits was repeated every third trial. Recall performance increased dramatically for the repeated sequence, in comparison with the nonrepeated sequences, although many of the participants were unaware of the repetition. This phenomenon is referred to as the Hebb repetition effect and has been classed as a form of implicit long-term learning (see, e.g., Seger, 1994).

There has been a reemergence of interest in the Hebb repetition effect with recent studies in which the mechanisms by which temporarily retained order information is translated into a more stable representation have been examined (see, e.g., Cumming, Page, \& Norris, 2003). The Hebb repetition effect has been tested primarily with verbal information, such as digits or letters in both auditory (e.g., Hebb, 1961) and visual (e.g., Hitch, Fastame, \& Flude, 2005) modalities. It has been suggested that long-

This work was supported in the form of a project grant to S.T. from the Natural Sciences and Engineering Research Council (NSERC) of Canada (CG073877). M.C. also receives support from NSERC. Part of this research was reported at the 44th Annual Meeting of the Psychonomic Society, November 2003, in Vancouver, Canada. We thank Dylan Jones, Robert Hughes, and François Vachon for critical readings of an earlier draft. Correspondence concerning this article should be addressed to S. Tremblay, École de Psychologie, Université Laval, Québec, PQ, G1K 7P4 Canada (e-mail: sebastien.tremblay@psy.ulaval.ca). term verbal sequence learning (including the Hebb effect) is driven by the action of the phonological loop (see, e.g., Baddeley, Gathercole, \& Papagno, 1998; Burgess \& Hitch, 1999; Cumming et al., 2003). Our purpose is to examine the Hebb repetition effect when the critical to-be-learned information consists of a sequence of visuospatial items and to establish whether verbal and spatial sequence learning share the same characteristics.

The characteristics of short-term serial recall are well established, and evidence of similarities between verbal and spatial information is accumulating (e.g., Jones, Farrand, Stuart, \& Morris, 1995; Ward, Avons, \& Melling, 2005). For example, serial position curves for serial spatial memory are strikingly similar to those obtained for serial verbal memory, exhibiting both marked primacy and recency effects (e.g., Avons, 1998; Farrand \& Jones, 1996; Nairne \& Dutta, 1992). Similarities are also observed in the pattern of errors found with verbal and spatial serial memory: An item is most often recalled near to its correct serial position (see Smyth \& Scholey, 1996). Other effects that have traditionally been associated with verbal shortterm memory (STM) occur in the spatial domain. First, the increase in the length of the to-be-remembered (TBR) list is associated with a decrease in performance (see Jones et al., 1995). Second, the mere presence of a to-be-ignored item at the end of the TBR list reduces recency for verbal, as well as spatial, information (Parmentier, Tremblay, \& Jones, 2004; see also Tremblay, Nicholls, Parmentier, \& Jones, 2005). Finally, it seems that both verbal and spatial recall performance benefit in similar ways from temporal grouping (Maybery, Parmentier, \& Jones, 2002). These examples suggest that processing serial order for spatial and verbal information is, at least in some key respects, functionally equivalent and that a Hebb repetition effect, similar to that traditionally obtained with verbal sequences, should also be observed in the visuospatial domain. 
There are some data from clinical and neuropsychological studies suggesting that the Hebb repetition effect can take place in the visuospatial domain. In what is probably the first demonstration of Hebb sequence learning with visuospatial sequences, Milner (1971), using a memory span procedure with the well-known Corsi block-tapping task to study hemispheric differences, observed a small but reliable effect of repetition (see also Corsi, 1972). With a similar procedure but a different objective - that of examining the impact of aging on learning for verbal and spatial sequences-Turcotte, Gagnon, and Poirier (2005) also found a Hebb repetition effect in the spatial domain. Although aging seemed to selectively diminish spatial learning, young adults showed learning of similar magnitude for both verbal and spatial sequences. However, in those studies, learning was inferred from the comparison between recall performance for random and repeated sequences averaged over trials (e.g., Milner, 1971) or divided into two blocks of trials (Turcotte et al., 2005), which restricts the analysis of the impact of repetitions (see Gagnon, Foster, Turcotte, \& Jongenelis, 2004, for a discussion).

One common criticism of the Corsi block task is that strategies based on verbal recoding (see Jones et al., 1995) or path configuration (see Berch, Krikorian, \& Huha, 1998) are likely to arise because the TBR objects are in a small matrix, there are often very few objects, their location is fixed, and all the objects remain visible throughout the presentation. Under such parameters, path configuration-organizing the TBR objects as geometric forms - is very likely (see Parmentier, Elford, \& Maybery, 2005, for a discussion). Although the Corsi block task has been widely used for clinical purposes over the last 30 years, the mechanisms underlying performance on this task are not yet well understood (Berch et al., 1998; see also Vandierendonck, Kemps, Fastame, \& Szmalec, 2004). It is unclear whether participants process the spatially distributed items in a serial fashion, as is the case with typical serial recall, and if so, whether participants associate each object with a digit label. Before concluding that the repetition of a sequence of visuospatial items presented for immediate serial recall leads to the long-term learning of that sequence, one has to rule out the possibility that the effect is mediated by verbal recoding or by path configuration strategies.

In order to test whether the Hebb repetition effect is obtained with visuospatial information and, hence, to explore further possible similarities between verbal and spatial memory/learning, a Hebb repetition manipulation was introduced into a visuospatial version of the immediate serial recall task, as previously used by Jones et al. (1995; see also Tremblay, Macken, \& Jones, 2001). In this task, a sequence of seven dots is presented on a computer monitor for immediate serial recall. A reconstruction method of recall is used in which, following presentation of the last dot, all seven dots are simultaneously re-presented in their original spatial locations and participants have to reproduce the order of presentation. The key manipulation introduced here, then, is that one series of dots was repeated every fourth trial. It is important to note that dots were presented one at a time $-700 \mathrm{msec}$ on and $300 \mathrm{msec}$ off-in order to reduce the possibility of the learning of path configurations.

In the present series of experiments, we wished to establish whether the Hebb repetition effect obtained with sequences of visuospatial items is functionally equivalent to that traditionally observed in the verbal domain (Hebb, 1961). That a Hebb repetition effect would be found in the visuospatial domain was a reasonable expectation for three reasons. First, a spatial Hebb repetition effect has been observed with the Corsi block task (e.g., Gagnon et al., 2004; Milner, 1971). Second, as was discussed, there are several similarities between the verbal and the spatial when serial order processing is required. Third, many studies have shown implicit sequence learning in the spatial domain where the task does not involve speech-based material. For example, in a serial reaction time (SRT) task, participants have to detect the onset of a target (e.g., an asterisk) presented in different locations on a monitor by pressing the key corresponding to a particular location (e.g., Nissen \& Bullemer, 1987; Remillard, 2003). When that stimulus follows some fixed repeated pattern, reaction time decreases significantly, in comparison with when the stimulus appears randomly (see Seger, 1994, for a review). In addition to replicating a Hebb effect in the spatial domain, we wish to examine whether some of the key characteristics of the verbal Hebb effect, such as the rate of learning over repetitions and the role of awareness, affect visuospatial material in a similar fashion. We also propose a simple method for measuring awareness and a novel approach with which to validate that measure.

A key issue related to the Hebb repetition effect in the context of implicit learning is whether participants are aware of the repetitions and whether that awareness has any consequence for the degree of learning. McKelvie (1987) reported that the awareness of the repeated series in the verbal domain had no impact on performance, since participants classified as aware and unaware exhibited similar learning curves (see also Stadler, 1993). A critical concern here is the ability of the measure of awareness to correctly classify participants. In Experiments 1 and 2, we opted to probe awareness by asking a simple question to the participants: "Did you notice anything particular about the procedure?" (taken from McKelvie's, 1987, questionnaire). Given the ambiguity of the latter question, it is unlikely to induce a false and retrospective impression of having been aware of the repetition during the experiment (see Sechler \& Watkins, 1991, for a discussion). The possibility that participants "become aware" when prompted about the repetition procedure is not always controlled for. In McKelvie's questionnaire, one example of a potentially biasing question when awareness is probed is, "Would you say that any set of digits was repeated during the experiment?" In order to validate our measure of awareness, we adopted the criteria of information and of sensitivity proposed by Shanks and St. John (1994). The criterion of information stipulates that in testing awareness, one must target the information that is responsible 
for the improved memory performance (i.e., in the present case, the repeated series), whereas the criterion of sensitivity refers to the probability that participants classified as aware explicitly retrieved that information. In order to meet these two criteria, we combined our simple probing question (used to classify participants as aware or unaware of the repetition) with a postexperimental recognition test for the repeated sequence (used to test explicit memory of the target information; see Eimer, Goschke, Schlaghecken, \& Stürmer, 1996, for a similar method employed in the context of SRT studies). It is expected that aware, rather than unaware, participants are likely to explicitly recognize the repeated sequence. This novel approach provides a validation of our measure of awareness: whether a simple ambiguous question is sensitive enough to detect participants aware of the repetition during the course of the experiment.

\section{EXPERIMENT 1}

The main objective of Experiment 1 was to reproduce the Hebb repetition effect in the visuospatial domain by using a variant of the Corsi block task developed by Jones et al. (1995). In this experiment, great care was taken to minimize the likelihood that the participants would verbally recode the TBR information phonologically, since the dots were in a different location every trial and guidelines such as grids were excluded. Also, as was mentioned above, the likelihood that a strategy based on path configuration will be used is reduced by employing a sequential presentation in which each dot appears and disappears before the next dot of the series.

\section{Method}

Participants. Forty students from Université Laval volunteered to take part in the experiment in exchange for a small honorarium. All reported normal or corrected-to-normal vision.

Materials. A sequence of seven black dots $1 \mathrm{~cm}$ in diameter was presented upon a white background on a PC computer screen, one dot at a time in a different location, within a $17 \times 17 \mathrm{~cm}$ frame. The coordinates for the dots' centers were randomly generated, with the constraint that the centers of all the dots, taken pairwise, should be separated by at least $3 \mathrm{~cm}$ and, at most, $10.5 \mathrm{~cm}$. On each trial, a sequence of seven dots was displayed at a rate of one dot every $1 \mathrm{sec}$ (700 $\mathrm{msec}$ on, $300 \mathrm{msec}$ off). Of the 50 sequences presented during the experimental session, 38 contained different arrangements of locations, and the other 12 sequences consisted of the repetition of the same arrangement in the same order. This repeated sequence varied across participants. Repetitions occurred at every fourth trial, with the first occurrence of the to-be-repeated sequence on Trial 4. The nonrepeated sequences were generated randomly, with the exception that no sequence could contain more than two dots that shared location and order with previous sequences. ${ }^{1}$ There were three nonrepeated (or random) trials before every repeated trial, and two nonrepeated trials followed the last repetition in order to avoid terminating the memory task with the repeated sequence.

Procedure. The participants were seated approximately $50 \mathrm{~cm}$ from the computer screen. The participants were told to remember all the items in the same order as that in which they appeared. Written instructions encouraged them to respond as quickly and accurately as possible and informed them that once an item had been selected, it would not be possible to alter it. The participants were informed that the purpose of the experiment was to evaluate visuospatial STM. Two practice trials were presented before the test proper began. The presentation of trials was self-paced; the participants mouse-clicked on a button on the screen to initiate a trial. Immediately following the presentation of the series, the seven dots were re-presented simultaneously in the same spatial locations as those in which they had originally been presented, which prompted the participants to start recalling them in order. The participants responded by using the mouse to locate and click on the dots in their original presentation order. To indicate that a response had been recorded, the color of a selected dot changed from black to green and remained that way until the end of the trial.

Following recall of the last sequence of dots, the participants were asked: "Did you notice anything particular about the procedure?" In order to be classified as aware, the participants had to meet a single criterion: They had to report anything that made reference to the fact that repetition had occurred during the experiment. After they had answered the latter question, the participants took part in a postexperimental recognition test. They were informed of the repetition procedure, but no information about the repeated sequence was given to them. For the recognition test, four dot sequences were presented, each within a frame, and were displayed in a $2 \times 2$ matrix on the computer screen. For each sequence, the seven dots were re-presented simultaneously. One of the four displays corresponded to the repeated sequence, and the other three were constructed randomly. The participants had to indicate which one of the four representations corresponded to the repeated sequence. No order information was provided on this task. The participants were tested individually, and the whole experimental session took $30 \mathrm{~min}$.

\section{Results}

For all of the experiments in this article, the participants' responses were scored according to the strict serial recall criterion: For the response to be scored correct, it had to correspond to the item's original serial position.

Gradients of improvement. The data for mean correct recall over repetitions (repeated sequence) and trials that preceded a repetition (nonrepeated sequences) are depicted in Figure 1A. It was important to make sure that the improvement in performance for the repeated sequence was greater than the one observed with the nonrepeated sequences, which would be associated with a general practice effect. In order to do so, gradients of improvement in number of correct responses, collapsed across serial positions, were calculated for each participant across the 12 repetitions and separately across the 12 groups of three matching nonrepeated trials. Using such a calculation provided us with individual slopes that reflected improvement over repetitions - that is, a fine-grained measure of the rate of learning (see, e.g., Cumming et al., 2003, and Hitch et al., 2005, for recent reports of gradients of improvement in the context of the Hebb effect for verbal materials). The mean improvement for the repeated sequence was .14 correct responses per repetition, as compared with .03 per presentation for the nonrepeated sequences. A $t$ test performed on these data showed the difference between repeated and nonrepeated to be significant $[t(39)=3.90$, $p<.005]$. One could argue that the improvement advantage for the repeated series was the result of the unusually low performance at its first occurrence (see Figure 1A). However, a $t$ test excluding the first presentation for both the repeated and the nonrepeated series showed that the 

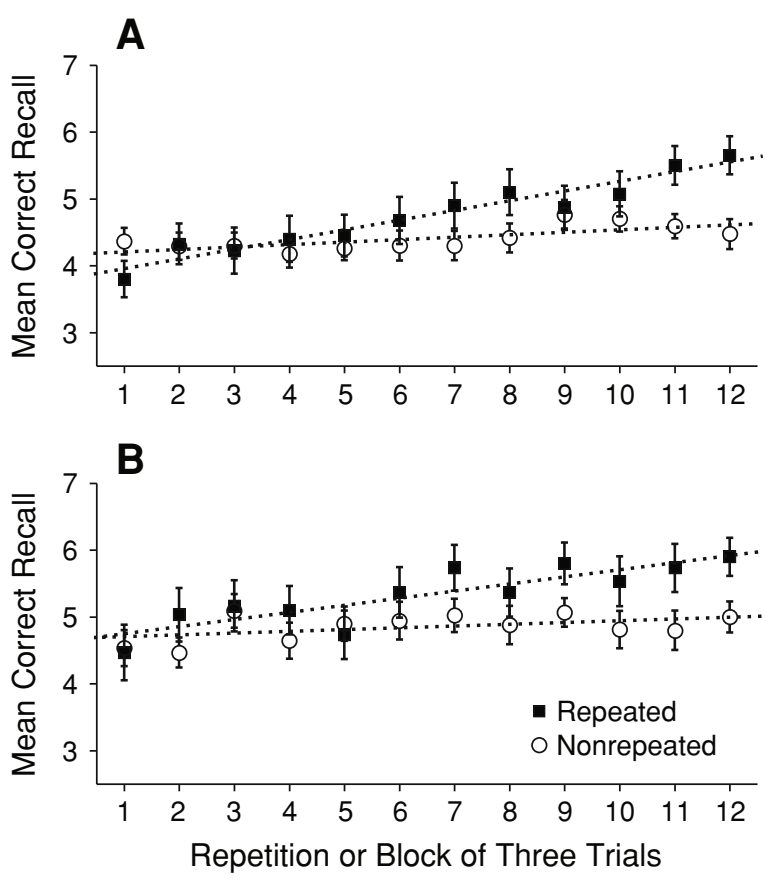

Figure 1. Mean correct recall for the Hebb repetitions and nonrepeated sequences (block of three trials) in (A) Experiment 1 and (B) Experiment 2. Regression lines are added to the plot.

improvement for the repeated series remains significantly greater $[t(39)=3.21, p<.005]$.

Serial position curves. Typical curves with primacy and recency were observed for both the repeated and the nonrepeated sequences (see Figure 2A). The data were also divided into blocks of trials (three blocks of 4 repeated trials and 12 matching nonrepeated trials) in order to provide an additional measure of the impact of repetitions on performance. A 3 (blocks) $\times 2$ (repetition: repeated or nonrepeated) $\times 7$ (serial position) ANOVA was performed on the proportion of correct serial recall. The main effects of serial position $[F(6,234)=55.27$, $p<.001]$ and of block $[F(2,78)=20.61, p<.001]$ were significant, whereas the main effect of repetition did not reach significance $[F(1,39)=2.43, p=.13]$. Most important, the interaction between block and repetition was significant $[F(2,78)=5.68, p<.01]$, which suggests that the difference between the repeated and the nonrepeated series varied over blocks of trials. The decomposition of the latter interaction showed that the difference between the repeated and the nonrepeated sequences reached significance only at Block $3[t(39)=2.38, p<.05]$. The difference between repeated and nonrepeated was not significant at Block $1[t(39)=-0.44, p=.664]$ or at Block $2[t(39)=1.73, p=.091]$. No other interactions were significant (all $\left.F_{\mathrm{S}}<1\right)$.

Degree of awareness. On the basis of the question the participants were asked, 19 of the 40 participants were classified as aware of repetition. The participants in the aware group performed better for the repeated sequence $[t(22)=5.56, p<.01]$, as well as for the nonrepeated sequences $[t(22)=6.12, p<.01]$. Their performance was greater than that of the unaware group from the very first occurrence of the to-be-repeated sequence onward (see Figure 3A). However, the observed learning curves were almost identical whether the participants were aware (mean improvement of .14 correct responses per repetition) or unaware (mean improvement of .15) of the repetition. A $t$ test performed on the gradients of improvement as a function of awareness revealed no difference between the aware and the unaware participants $[t(38)=-0.17$, $p=.866]$.

Sensitivity of our measure of awareness was calculated by dividing the number of aware participants - those who reported having noticed the repetition-who provided the correct response at the recognition test by the total number of aware participants. Eighteen of the 19 aware participants provided us with the correct answer at the recognition test. Taking into account performance at the recognition test, the sensitivity of the question employed to probe awareness - its ability to classify correctly aware participants as aware — was estimated at $94.74 \%$.

\section{Discussion}

The pattern of results confirms that a Hebb repetition effect occurs with visuospatial sequences. Repetition of the same sequence of dots on every fourth trial led to a marked improvement in recall performance, in comparison with nonrepeated random sequences. As is the case in the verbal domain, this visuospatial Hebb repetition effect seems to be independent of awareness, since the participants aware of the repetition showed no extra gain in performance over repetitions (see also McKelvie, 1987). Although the performance of the aware participants was better on every trial than that of the unaware participants, there was no difference between the aware and the unaware participants in terms of the improvement over repetitions of the Hebb list. The latter finding rules out the possibility that learning was due merely to being aware of the repetition and, thus, was the result of explicit mechanisms. Moreover, using a postexperimental recognition test combined with the measure of sensitivity provided us with an objective estimation of the extent to which the aware participants were correctly classified.

\section{EXPERIMENT 2}

In Experiment 1, we showed a marked effect of repetition, using the typical dot task in which verbal recoding is very unlikely. Indeed, for lists other than the repeating list, the locations of the dots were different for every trial, as was the order of presentation (see Jones et al., 1995, for a discussion). Thus, in Experiment 1, the repeated sequence differed from the nonrepeated series in terms of both item and order information (to minimize a possible verbal recoding). However, in the original procedure with verbal information, a fixed set of items was used. One could argue that the Hebb repetition effect found in Experiment 1 was qualitatively different from the verbal variant, in that it may have been driven by the repetition of item 

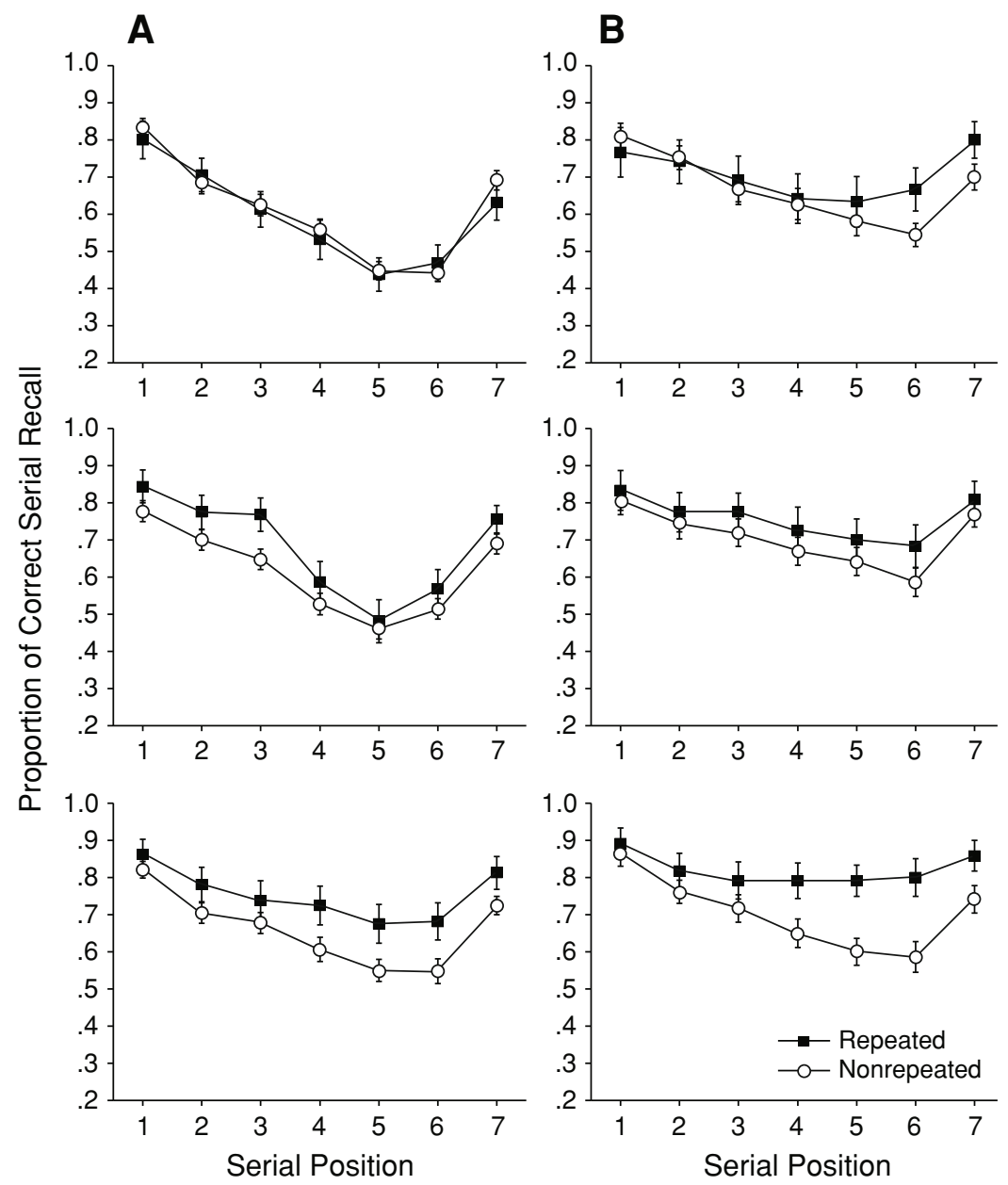

Figure 2. Proportion of correct serial recall as a function of serial position for repeated and nonrepeated sequences in (A) Experiment 1 and (B) Experiment 2 for Blocks 1 (upper panel), 2 (middle panel), and 3 (lower panel). Error bars represent standard errors.

information (dot locations), rather than by the particular order of dot locations. Also, it seems that the degree of similarity between repeated and nonrepeated sequences can influence the rate of learning. Indeed, in the verbal domain, Melton (1967) showed that when the repeated series differed from the nonrepeated ones in terms of both order and item information, the learning curve was significantly steeper than when the sequences differed only in terms of order information. Thus, in Experiment 2, the same fixed set of seven dots was used for each trial (but here, also, we employed a typical serial memory procedure with a sequential presentation of the dots - on and off - and no gridlines, in order to minimize the use of verbal recoding or path configuration strategies).

\section{Method}

Participants. Thirty students from Université Laval took part in Experiment 2 in exchange for a small fee. All reported normal or corrected-to-normal vision. None of the participants had taken part in Experiment 1.
Materials. The method in Experiment 2 was identical to that in Experiment 1, except that the same seven locations were used to display the dots; only the order in which the dots were presented varied across repeated and nonrepeated trials. However, it is important to note that each participant was presented with a different set of dot locations.

The design and procedure were identical to those employed in Experiment 1. Because the item information was the same for each trial, the postexperimental recognition test required the participants to recognize the correct sequence in which the items of the repeated sequences had been presented. The participants were presented with four schematic representations of the dots, in which labels indicated their order of appearance in the sequence. They had to select the correct representation.

\section{Results}

Gradients of improvement. The mean improvement for the repeated sequence was .11 correct responses per repetition, as compared with .03 per presentation for the nonrepeated sequences (see Figure 1B). A $t$ test performed on these data showed the difference between repeated and 


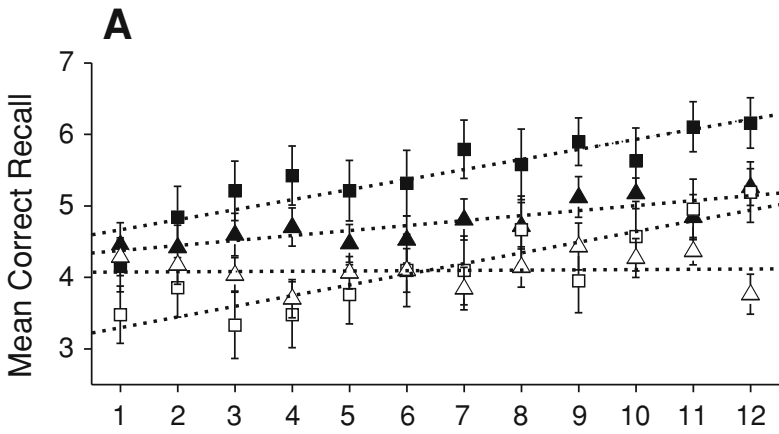

B

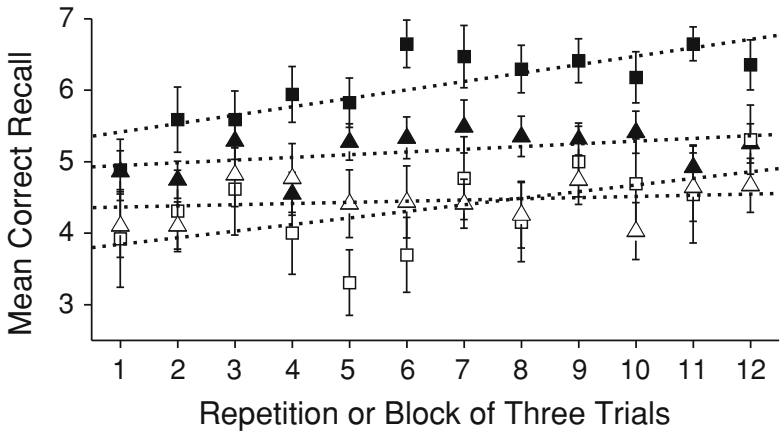

Aware repeated

- Aware nonrepeated

$\square$ Unaware repeated

$\triangle$ Unaware nonrepeated

Figure 3. Mean correct recall for aware and unaware participants as a function of repetition (repeated series) or block of three trials (nonrepeated series) for (A) Experiment 1 and (B) Experiment 2. Regression lines are added to the plot.

nonrepeated to be significant $[t(29)=2.51, p<.05]$. A $t$ test carried out on the repeated sequence data for Experiment 1 and Experiment 2 showed that the gradients of improvement were not significantly different $[t(68)=$ $1.07, p=.29$ ], although numerically, the mean gradient obtained in Experiment 1 was higher (.14).

Serial position curves. Typical curves were observed for both the repeated and the nonrepeated sequences (see Figure 2B). As in Experiment 1, a $3 \times 2 \times 7$ ANOVA was performed on the proportion of correct serial recall. The main effects of block $[F(2,58)=5.43, p=.014]$, serial position $[F(6,174)=13.63, p<.001]$, and repetition $[F(1,29)=9.18, p<.01]$ were significant and so was the interaction between block and repetition $[F(2,58)=3.33$, $p<.05]$. The decomposition of this interaction showed that learning, as in Experiment 1, took place in Block 3 $[t(29)=4.69, p<.01]$. The difference between the repeated and the nonrepeated sequences was not significant at Block $1[t(29)=1.02, p=.317]$ or Block $2[t(29)=$ $1.96, p=.060]$.

Degree of awareness. Eighteen participants were classified as aware of repetition, and 12 as unaware. Again, the aware participants showed greater performance for the repeated sequence $[t(28)=3.85, p<.01]$, as well as for the nonrepeated series $[t(28)=1.93, p=.05]$. However, as in Experiment 1, mean improvement gradients for the repeated series were similar for the aware (.12 correct responses per repetition) and the unaware (.09 correct responses per repetition) participants. A $t$ test performed on these gradients revealed no difference between the aware and the unaware participants $[t(28)=0.41, p=.68$; see Figure 3B]. Since the same seven dots were presented on each trial, the recognition test relied on order information only. Out of 18 aware participants, 15 provided us with the correct recognition, which gives a sensitivity of $83.33 \%$.

\section{Discussion}

A Hebb repetition effect was found in the second experiment when the repeated sequence differed from the nonrepeated sequences only in terms of order information. As was the case in Experiment 1, the unaware participants showed an improvement similar to that of the aware participants. Although there was a numerical trend for the dissimilar repeated and nonrepeated sequences (distinct on both order and item dimensions in Experiment 1) to produce a greater rate of learning than did the similar sequences (Experiment 2), the difference was not statistically significant. Statistically, this result does not replicate the one obtained by Melton (1967), who showed a greater learning rate for sequences that differed in terms of both order and item information with verbal material. However, one possible explanation for the small nonsignificant effect of similarity is concerned with the nature of the TBR stimuli. The characteristics on which repeated and nonrepeated series differed are difficult to equate between verbal and visuospatial sequences. Indeed, dissimilarity in the verbal domain was made up of nine consonants in a fixed order for the repeated sequence and nine conso- 
nants in random orders for the nonrepeated sequences. Manipulation of similarity in Experiment 2 relied on always presenting the same seven dot locations for the repeated sequence and all-different dot locations for the nonrepeated sequences. It is plausible that discrimination between repeated and nonrepeated sequences was more obvious in the verbal domain.

\section{EXPERIMENT 3}

Both Experiments 1 and 2 provided further evidence that the Hebb repetition effect can take place when the TBR information is visuospatial (see also Milner, 1971; Turcotte et al., 2005). However, a comparison of verbal and spatial Hebb effects across studies raises problems. It would be difficult to carry out a reliable meta-analysis to compare these effects because of the numerous methodological differences across studies. For example, in some studies, a memory set of nine digits has been used (e.g., Hebb, 1961; McKelvie, 1987), and in others, a set of eight letters (e.g., Cunningham, Healy, \& Williams, 1984). There is apparently no study in which the magnitude of the Hebb repetition effect and its learning curves have been compared directly for verbal and visuospatial sequences. The purpose of the third experiment was to compare the Hebb repetition effects in verbal and visuospatial domains by using a within-subjects design and making sure that the verbal and the visuospatial tasks were as similar as possible.

\section{Method}

Participants. Twenty-four students from Université Laval volunteered to take part in the experiment in exchange for a small honorarium. All reported normal or corrected-to-normal vision. None of the participants had taken part in Experiment 1 or 2.

Materials. For the visuospatial task, the method in Experiment 3 was identical to that in Experiment 2, except that nine dots were displayed in each sequence, instead of seven. The same nine locations were used to display the dots; only the order in which the dots were presented varied across repeated and nonrepeated trials. Each participant was presented with a different set of dot locations. For the auditory verbal task, nine letters ( $\mathrm{D}-\mathrm{F}-\mathrm{G}-\mathrm{H}-\mathrm{K}-\mathrm{L}-\mathrm{N}-\mathrm{Q}-\mathrm{R})$ were recorded in a female voice. Each sequence was played at a rate of one letter per second through headphones (700 msec on, $300 \mathrm{msec}$ off) at approximately $65 \mathrm{db}(\mathrm{A})$. After the presentation of each sequence, the nine letters were randomly presented on the computer screen in a horizontal fashion (at the center of a $17 \times 17 \mathrm{~cm}$ frame).

Procedure. The procedure was similar to the one used in Experiments 1 and 2. Each participant took part in both the auditory-verbal and the visuospatial tasks. Half of the participants began with the auditory-verbal task, and the other half, with the visuospatial task. There was no postexperimental session and no measure of awareness, since it would have been difficult to ascribe awareness to either the auditory-verbal or the visuospatial task. The participants were tested individually, and the experiment took $60 \mathrm{~min}$.

\section{Results and Discussion}

Gradients of improvement. The mean improvement for the repeated sequence was .15 correct responses per repetition in the verbal domain (.05 for the nonrepeated sequences), as compared with .17 correct responses per repetition with the visuospatial sequences $(-.02$ for the

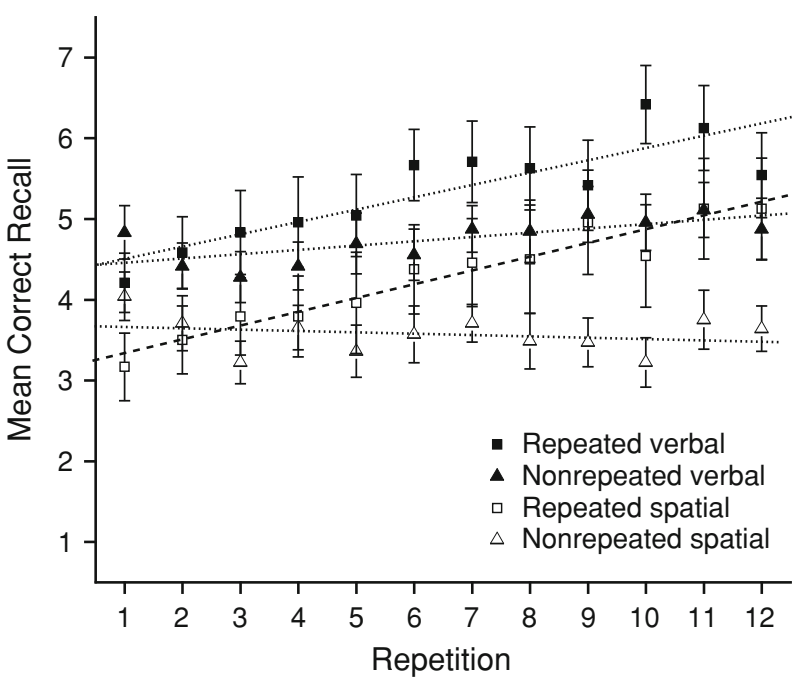

Figure 4. Mean correct recall for verbal and spatial series as a function of repetition (repeated series) or block of three trials (nonrepeated series). Regression lines are added to the plot.

nonrepeated sequences; see Figure 4). A 2 (repetition: repeated or nonrepeated) $\times 2$ (type: verbal or spatial) ANOVA was performed on these data. The main effect of repetition was significant $[F(1,22)=9.52, p<.01]$, but the difference between verbal and spatial was not significant $(F<1)$, nor was the interaction of type and repetition $[F(1,22)=1.81, p=.192]$. This pattern of results shows that the Hebb repetition effects found in the verbal and the spatial domains are functionally equivalent in relation to the rate of learning.

Serial position curves. Typical curves were observed for both the repeated and the nonrepeated sequences with auditory-verbal and visuospatial material (see Figure 5). A 2 (type) $\times 2$ (repetition) $\times 3$ (blocks) $\times 7$ (serial position) ANOVA was performed on the proportion of correct serial recall. All the main effects [type, $F(1,23)=10.55$, $p<.01$; blocks, $F(2,46)=16.62, p<.001$; repetition, $F(1,23)=10.04, p<.01$; and serial position, $F(8,184)=$ $39.30, p<.001]$ were significant. For the sake of brevity, only the interactions most relevant to the issues at test will be reported here. Importantly, the interaction between type and repetition was not significant $(F<1)$, whereas the interaction of block and repetition $[F(2,46)=6.45$, $p<.01]$ was significant. The latter interaction did not vary as a function of type $[F(2,46)=1.19, p<.31]$. All other three-way interactions and the four-way interaction were not significant (all $p \mathrm{~s}>.40$ ). The present pattern of significance clearly indicates that sequence learning (as well as the serial position curves) is very similar whether the information is verbal or spatial.

\section{GENERAL DISCUSSION}

In Experiment 1, in which there was little possibility of verbal recoding, we found a Hebb repetition effect with spatial stimuli. This visuospatial Hebb effect was 

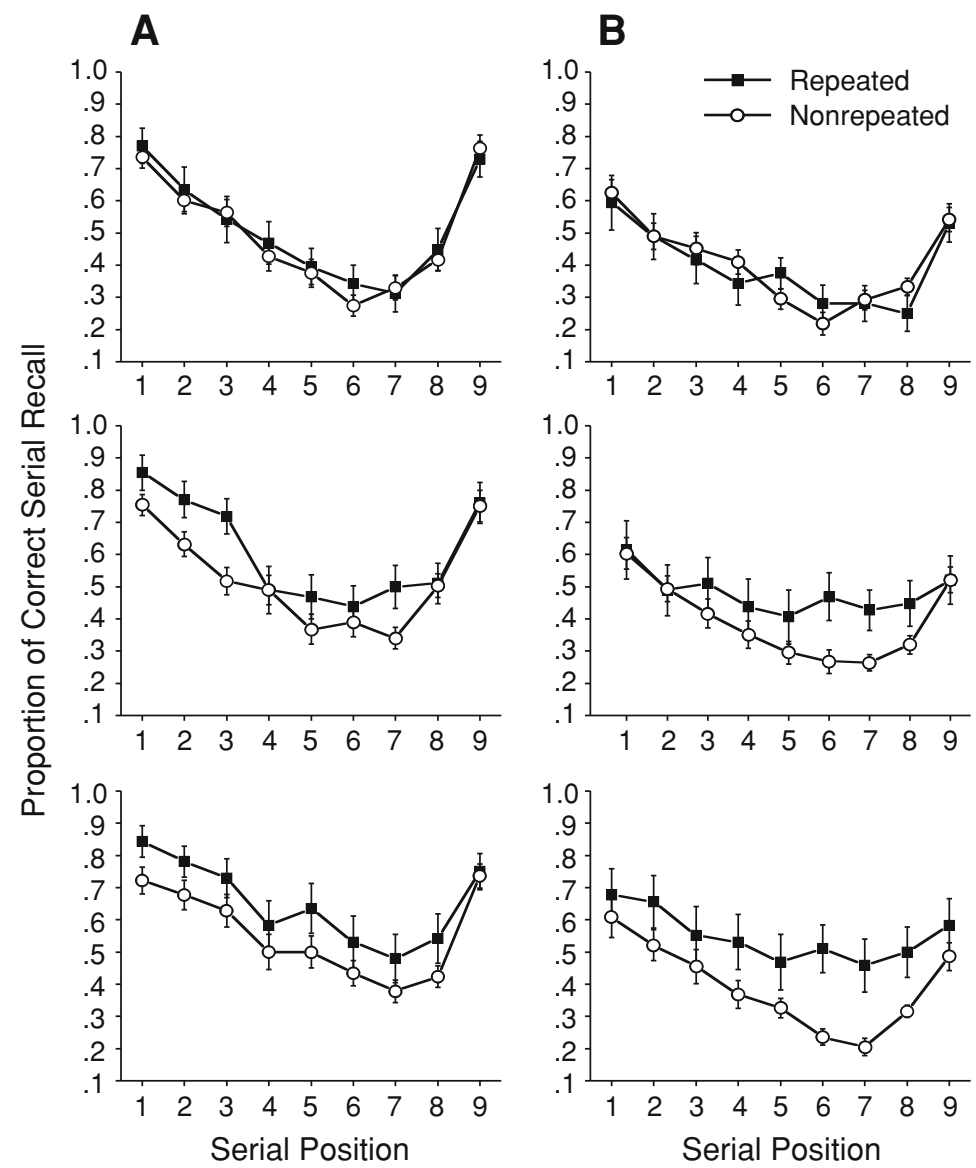

Figure 5. Proportion of correct serial recall as a function of type of series (repeated or nonrepeated) and serial position for (A) verbal series and (B) spatial series for Blocks 1 (upper panel), 2 (middle panel), and 3 (lower panel). Error bars represent standard errors.

also obtained when the repeated sequence differed only in terms of order information, as in the original procedure with verbal information. The comparison of the learning curve obtained in Experiment 1 with that observed in Experiment 2 is inconclusive in showing that the degree of similarity between repeated and nonrepeated sequences can modulate the rate of learning in the spatial domain as in the verbal domain (see Melton, 1967). The results of Experiments 1 and 2 are consistent with McKelvie's (1987) conclusion that the Hebb effect reflects a form of implicit learning: Even though the performance of the aware participants was higher than that of the unaware participants on each trial, both exhibited similar learning curves. In Experiment 3, a direct comparison, within a repeated measures design, between Hebb learning in the auditory-verbal and the visuospatial domains provides unequivocal evidence that the rate of learning due to Hebb repetitions is the same regardless of the nature of the stimuli. Thus, our results provide further evidence that the Hebb repetition effect extends to visuospatial information and that its basic characteristics seem functionally similar to those found in the verbal literature (e.g., Hebb,
1961; McKelvie, 1987; Melton, 1963; Sechler \& Watkins, 1991). It is important to mention that our results suggest that the measure of awareness can be very simple and yet meet all the criteria for a reliable test of such awareness (Shanks \& St. John, 1994). Indeed, our method relies on a single question, and its combination with a recognition test has provided evidence that the measure is sensitive and discriminating.

The unequivocal demonstration of similar Hebb repetition learning for verbal and spatial sequences begs the question of what exactly is being learned over repetitions. Although it is clear that the learning substratum is not speech based, the nature of the abstract representation being formed over repetitions remains uncertain. In the present series of experiments, it is possible that what was learned was the motor response pattern associated with the repeated sequence. However, there is ample evidence that learning of repeated information does not require participants to reproduce the sequence over its repetitions (e.g., Cunningham et al., 1984; Remillard, 2003). One possibility is that relationships among items, rather than item information per se, are at the basis of what might 
be learned. Indeed, given the serial nature of the memory task, it is unlikely that the product of learning takes the form of some item-based or configural representation.

A key theoretical question related to the latter issue is what drives the Hebb repetition effect. Some authors have suggested that the effect is the consequence of rehearsal processes (e.g., Cunningham et al., 1984; see also Burgess \& Hitch, 1999) or output processes (e.g., Cohen \& Johansson, 1967). Other studies have proposed that learning of repeated information can take place at a perceptual stage - that is, even when rehearsal and responses are proscribed (e.g., Remillard, 2003). The visuospatial Hebb effect found in the present study casts further doubt on a phonological-loop-based account of verbal long-term sequence learning: The learning mechanism clearly transcends the content of the to-be-learned material (see, e.g., Saffran, Johnson, Aslin, \& Newport, 1999).

Some computational models of how serial order is stored in memory eschew the fractionation of STM processing into distinct modules, whereas others offer what seem to be amodal mechanisms of processing order but are underpinned by the working memory modular architecture (e.g., Burgess \& Hitch, 1999; Page \& Norris, 1998). Most computational models suggest that order representation in memory is a result of context associations between items (chaining models; see, e.g., Lewandowski \& Murdock, 1989; Murdock, 1995) or between positions and items (positional models; see, e.g., Burgess \& Hitch, 1999; Lee \& Estes, 1981; see also Brown, Preece, \& Hulme, 2000, for a time-based associative model). A prediction common to these models is that with the repetition of the series, such associations are reinforced and, thus, lead to the improvement of serial recall performance. However, there is some evidence that the strengthening of item position or time-based associations cannot account for the Hebb repetition effect. For example, Cumming et al. (2003) showed that when the items of the repeated list were displaced in a final transfer list, there was no improvement either for the items that kept their serial position or for the items that changed their serial position, in comparison with control lists in which the whole sequence remained intact. Thus, it seems that interitem relationships play at least some role in the Hebb effect (see Hitch et al., 2005, for a discussion; see also Schwartz \& Bryden, 1971). Cumming et al. put forward the idea that as a sequence is repeated, some cumulative chunking process occurs, thereby making the repeated list easier to serially recall (see Page \& Norris, 1998). However, again, in the latter account of the Hebb effect, a predominant role is attributed to a phonological component, in that the action of the chunking mechanism is claimed to take place in a speech-based store.

There is substantial divergence among researchers in explaining how serial order is stored in memory and what factors underpin the Hebb repetition effect. One consideration that stems from our results is that models must take into account that the Hebb effect is not restricted to speech-based material. That the repetition of a visuospatial sequence in a serial recall paradigm produces the same long-term learning effect as the one that occurs for a repeating verbal sequence suggests that the learning mechanism is not uniquely verbal and transcends the content of what is to be learned. If the learning mechanism underpinning the Hebb effect is not a mechanism peculiar to verbal input, the notion that the verbal Hebb effect reflects the action of a dedicated verbal (or phonological) learning device begins to look unparsimonious. In conclusion, it can be argued that exploring the characteristics of the Hebb repetition effect in the verbal and spatial domains may provide useful insights into modeling both explicit and implicit sequence learning and may inform the debate concerning the extent to which serial order for verbal and spatial information is processed in a functionally similar fashion.

\section{REFERENCES}

Avons, S. E. (1998). Serial report and item recognition of novel visual patterns. British Journal of Psychology, 89, 285-308.

Baddeley, A., Gathercole, S., \& Papagno, C. (1998). The phonological loop as a language learning device. Psychological Review, 105, 158-173.

BerCH, D. B., Krikorian, R., \& Huha, E. M. (1998). The Corsi blocktapping task: Methodological and theoretical considerations. Brain \& Cognition, 38, 317-338.

Brown, G. D. A., Preece, T., \& Hulme, C. (2000). Oscillator-based memory for serial order. Psychological Review, 107, 127-181.

Burgess, N., \& Hitch, G. J. (1999). Memory for serial order: A network model of the phonological loop and its timing. Psychological Review, 106, 551-581.

CoHEn, R. L., \& Johansson, B. S. (1967). The activity trace in immediate memory: A re-evaluation. Journal of Verbal Learning \& Verbal Behavior, 6, 139-143.

Consi, P. (1972). Human memory and the medial temporal region of the brain. Unpublished doctoral thesis, McGill University.

Cumming, N., Page, M., \& Norris, D. (2003). Testing a positional model of the Hebb effect. Memory, 11, 43-63.

Cunningham, T. F., Healy, A. F., \& Williams, D. M. (1984). Effects of repetition on short-term retention of order information. Journal of Experimental Psychology: Learning, Memory, \& Cognition, 10, 575-597.

Eimer, M., Goschke, T., Schlaghecken, F., \& Stürmer, B. (1996). Explicit and implicit learning of event sequences: Evidence from event-related brain potentials. Journal of Experimental Psychology: Learning, Memory, \& Cognition, 22, 970-987.

FARRAND, P., \& JONES, D. (1996). Direction of report in spatial and verbal serial short-term memory. Quarterly Journal of Experimental Psychology, 49A, 140-158.

Gagnon, S., Foster, J. K., Turcotte, J., \& Jongenelis, S. (2004). Involvement of the hippocampus in implicit learning of supra-span sequences: The case of SJ. Cognitive Neuropsychology, 21, 867-882.

HebB, D. O. (1961). Distinctive features of learning in the higher animal. In J. F. Delafresnaye (Ed.), Brain mechanisms and learning (pp. 3746). Oxford: Blackwell.

Hitch, G. J., Fastame, M. C., \& Flude, B. (2005). How is the serial order of a verbal sequence coded? Some comparisons between models. Memory, 13, 247-258.

Jones, D., Farrand, P., Stuart, G., \& Morris, N. (1995). Functional equivalence of verbal and spatial information in serial short-term memory. Journal of Experimental Psychology: Learning, Memory, \& Cognition, 21, 1008-1018.

LASHLEY, K. S. (1951). The problem of serial order in behavior. In L. A. Jeffress (Ed.), Cerebral mechanisms in behavior: The Hixon Symposium (pp. 112-136). New York: Wiley.

LEE, C. L., \& Estes, W. K. (1981). Item and order information in shortterm memory: Evidence for multilevel perturbation processes. Jour- 
nal of Experimental Psychology: Human Learning \& Memory, 7, 149-169.

Lewandowsky, S., \& Murdock, B. B., Jr. (1989). Memory for serial order. Psychological Review, 96, 25-57.

Maybery, M. T., Parmentier, F. B. R., \& Jones, D. M. (2002). Grouping of list items reflected in the timing of recall: Implications for models of serial verbal memory. Journal of Memory \& Language, 47, 360-385.

MCKELVIE, S. J. (1987). Learning and awareness in the Hebb digits task. Journal of General Psychology, 114, 75-88.

Melton, A. W. (1963). Implications of short-term memory for a general theory of memory. Journal of Verbal Learning \& Verbal Behavior, 2, $1-21$.

MELTON, A. W. (1967). Relations between short-term memory for a general theory of memory. In D. P. Kimble (Ed.), The organization of recall: Proceedings of the second International Interdisciplinary Conference on Learning, Remembering, and Forgetting (Vol. 2, pp. 2462). New York: New York Academy of Sciences, Interdisciplinary Communications Program.

MiLNER, B. (1971). Interhemispheric differences in the localization of psychological processes in man. British Medical Bulletin, 27, 272277.

MURDOCK, B. B. (1995). Developing TODAM: Three models for serialorder information. Memory \& Cognition, 23, 631-645.

NAIRNE, J. S., \& DutTA, A. (1992). Spatial and temporal uncertainty in long-term memory. Journal of Memory \& Language, 31, 396-407.

Nissen, M. J., \& Bullemer, P. (1987). Attentional requirements of learning: Evidence from performance measures. Cognitive Psychology, 19, 1-32

PAGE, M. P. A., \& Norris, D. (1998). The primacy model: A new model of immediate serial recall. Psychological Review, 105, 761-781.

Parmentier, F. B. R., Elford, G., \& Maybery, M. (2005). Transitional information in spatial serial memory: Path characteristics affect recall performance. Journal of Experimental Psychology: Learning, Memory, \& Cognition, 31, 412-427.

Parmentier, F. B. R., Tremblay, S., \& Jones, D. M. (2004). Exploring the suffix effect in serial visuospatial short-term memory. Psychonomic Bulletin \& Review, 11, 289-295.

REMILLARD, G. (2003). Pure perceptual-based sequence learning. Journal of Experimental Psychology: Learning, Memory, \& Cognition, 29, 581-597.

SAFFrAn, J. R., JohnSon, E. K., Aslin, R. N., \& Newport, E. L. (1999). Statistical learning of tone sequences by human infants and adults. Cognition, 70, 27-52.
Schwartz, M., \& Bryden, M. P. (1971). Coding factors in the learning of repeated digit sequences. Journal of Experimental Psychology, 87, 331-334.

Sechler, E. S., \& Watkins, M. J. (1991). Learning to reproduce a list and memory for the learning. American Journal of Psychology, 104, 367-394.

SEGER, C. A. (1994). Implicit learning. Psychological Bulletin, 115, 163-196.

SHANKS, D. R., \& ST. JoHN, M. F. (1994). Characteristics of dissociable human learning systems. Behavioral \& Brain Sciences, 17, 367-447.

Smyth, M. M., \& Scholey, K. A. (1996). Serial order in spatial immediate memory. Quarterly Journal of Experimental Psychology, 49A, 159-177.

StadLer, M. A. (1993). Implicit serial learning: Questions inspired by Hebb (1961). Memory \& Cognition, 21, 819-827.

Tremblay, S., Macken, W. J., \& Jones, D. M. (2001). The impact of broadband noise on serial memory: Changes in band-pass frequency increase disruption. Memory, 9, 323-331.

Tremblay, S., Nichol LS, A. P., Parmentier, F. B. R., \& Jones, D. M. (2005). Visual distraction and visuo-spatial memory: A sandwich effect. Memory, 13, 357-363.

Turcotte, J., Gagnon, S., \& Poirier, M. (2005). The effect of old age on the learning of supraspan sequences. Psychology \& Aging, 20, 251-260.

Vandierendonck, A., Kemps, E., Fastame, M. C., \& Szmalec, A. (2004). Working memory components of the Corsi blocks task. British Journal of Psychology, 95, 57-79.

WARD, G., Avons, S. E., \& MELLING, L. (2005). Serial position curves in short-term memory: Functional equivalence across modalities. Memory, 13, 308-317.

\section{NOTE}

1. Here and elsewhere in the series of experiments, the spatial characteristics of the dot sequences, such as path length - the distance between two successive dots - and number of crossings in the path sequence, are not systematically equated for repeated and nonrepeated sequences. Although path length and number of crossings have been shown to affect serial memory performance (see Parmentier et al., 2005), the random generation of dot sequences across trials and participants reduces the risk of contamination.

(Manuscript received February 7, 2005; revision accepted for publication September 17, 2005.) 